Acta Crystallographica Section E

Structure Reports

Online

ISSN 1600-5368

\section{Acridinium 2-hydroxybenzoate}

\section{Hossein Eshtiagh-Hosseini, ${ }^{*}$ Azam Hassanpoor, Masoud Mirzaei and Ali R. Salimi}

Department of Chemistry, School of Sciences, Ferdowsi University of Mashhad,

Mashhad, Iran

Correspondence e-mail: heshtiagh@ferdowsi.um.ac.ir

Received 26 September 2010; accepted 25 October 2010

Key indicators: single-crystal X-ray study; $T=100 \mathrm{~K}$; mean $\sigma(\mathrm{C}-\mathrm{C})=0.002 \AA$; $R$ factor $=0.048 ; w R$ factor $=0.128 ;$ data-to-parameter ratio $=18.6$.

In the title compound, $\mathrm{C}_{13} \mathrm{H}_{10} \mathrm{~N}^{+} \cdot \mathrm{C}_{7} \mathrm{H}_{5} \mathrm{O}_{3}{ }^{-}$or $(\mathrm{acrH})^{+}(\mathrm{Hsal})^{-}$, the asymmetric unit contains one acridinium cation and one salicylate anion. The acridinium $\mathrm{N}$ atom is protonated and the carboxylic acid group of salicylic acid is deprotonated. Both moieties are planar, with an r.m.s. deviation of $0.0127 \AA$ for the acr cation and $0.0235^{\circ}$ for the sal anion. They are aligned with a dihedral angle of $71.68(3)^{\circ}$ between them. The crystal structure is stabilized by a network of intermolecular $\mathrm{N}-$ $\mathrm{H} \cdots \mathrm{O}, \mathrm{O}-\mathrm{H} \cdots \mathrm{O}$ and $\mathrm{C}-\mathrm{H} \cdots \mathrm{O}$ hydrogen bonds. $\mathrm{C}-\mathrm{H} \cdots \pi$ interactions are also present.

\section{Related literature}

For work on molecular self-association, see: Moghimi et al. (2005); Eshtiagh-Hosseini, Hassanpoor, Canadillas-Delgado \& Mirzaei (2010); Eshtiagh-Hosseini, Mahjoobizadeh \& Mirzaei (2010). For related structures, see: Gellert \& Hsu (1988); Hemamalini \& Fun (2010); Muthiah et al. (2006).

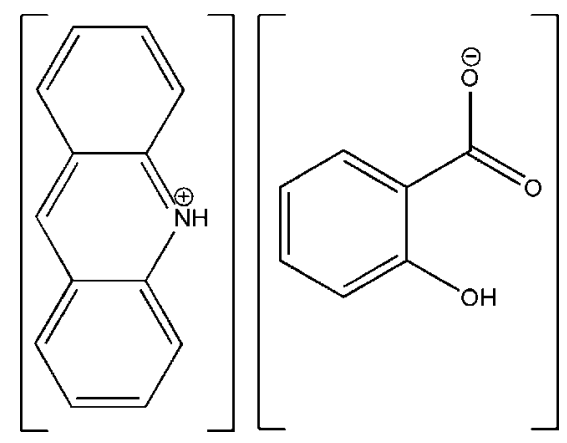

\section{Experimental}

\section{Crystal data}

$\mathrm{C}_{13} \mathrm{H}_{10} \mathrm{~N}^{+} \cdot \mathrm{C}_{7} \mathrm{H}_{5} \mathrm{O}_{3}^{-}$

$M_{r}=317.33$

Monoclinic, $P 2_{1} / c$ $\beta=91.449(10)^{\circ}$

$V=1527.9(10) \AA^{3}$

$Z=4$

Mo $K \alpha$ radiation

$\mu=0.09 \mathrm{~mm}^{-1}$

$T=100 \mathrm{~K}$

$0.30 \times 0.25 \times 0.10 \mathrm{~mm}$

\section{Data collection}

Bruker SMART APEXII CCD area-detector diffractometer

Absorption correction: multi-scan (SADABS; Bruker, 2001)

$T_{\min }=0.973, T_{\max }=0.991$

Refinement

$R\left[F^{2}>2 \sigma\left(F^{2}\right)\right]=0.048$

$w R\left(F^{2}\right)=0.128$

$S=1.04$

4488 reflections

241 parameters

10437 measured reflections 4488 independent reflections 3161 reflections with $I>2 \sigma(I)$ $R_{\text {int }}=0.035$

$\mathrm{H}$ atoms treated by a mixture of independent and constrained refinement

$\Delta \rho_{\max }=0.37{\mathrm{e} \AA^{-3}}^{-3}$

$\Delta \rho_{\min }=-0.24$ e $\AA^{-3}$

Table 1

Hydrogen-bond geometry $\left(\AA,^{\circ}\right)$.

$\mathrm{Cg} 1$ is the centroid of the $\mathrm{C} 2-\mathrm{C} 7$ benzene ring of $\mathrm{Hsal}^{-}$.

\begin{tabular}{lllll}
\hline$D-\mathrm{H} \cdots A$ & $D-\mathrm{H}$ & $\mathrm{H} \cdots A$ & $D \cdots A$ & $D-\mathrm{H} \cdots A$ \\
\hline $\mathrm{N} 1-\mathrm{H} 1 \cdots \mathrm{O} 1^{\mathrm{i}}$ & $1.05(2)$ & $2.49(2)$ & $3.100(2)$ & $116.4(15)$ \\
$\mathrm{N} 1-\mathrm{H} 1 \cdots \mathrm{O} 2^{\mathrm{i}}$ & $1.05(2)$ & $1.55(2)$ & $2.5887(19)$ & $174.8(18)$ \\
$\mathrm{O} 3-\mathrm{H} 3 \cdots \mathrm{O} 1$ & $1.00(3)$ & $1.58(2)$ & $2.5141(19)$ & $153(2)$ \\
$\mathrm{C} 10-\mathrm{H} 10 \cdots \mathrm{O} 1^{\text {ii }}$ & 0.93 & 2.49 & $3.294(2)$ & 145 \\
$\mathrm{C} 18-\mathrm{H} 18 \cdots \mathrm{O}{ }^{\text {iii }}$ & 0.93 & 2.46 & $3.135(2)$ & 129 \\
$\mathrm{C} 14-\mathrm{H} 14 \cdots C g 1^{\text {iv }}$ & 0.93 & 2.76 & $3.644(2)$ & 159 \\
$\mathrm{C} 17-\mathrm{H} 17 \cdots C g 1$ & 0.93 & 2.91 & $3.716(2)$ & 146 \\
\hline
\end{tabular}

Symmetry codes: (i) $\quad x,-y+\frac{1}{2}, z-\frac{1}{2}$; $\quad$ (ii) $\quad-x+2, y+\frac{1}{2},-z+\frac{1}{2}$; $-x+2, y-\frac{1}{2},-z+\frac{1}{2}$; (iv) $x,-y+\frac{3}{2}, z-\frac{1}{2}$.

Data collection: APEX2 (Bruker, 2005); cell refinement: SAINT (Bruker, 2005); data reduction: $S A I N T$; $\operatorname{program}(\mathrm{s})$ used to solve structure: SHELXS97 (Sheldrick, 2008); program(s) used to refine structure: SHELXL97 (Sheldrick, 2008); molecular graphics: ORTEP-3 for Windows (Farrugia, 1997); software used to prepare material for publication: Win $G X$ (Farrugia, 1999).

The Ferdowsi University of Mashhad is gratefully acknowledged for financial support.

Supplementary data and figures for this paper are available from the IUCr electronic archives (Reference: BQ2237).

\section{References}

Bruker (2001). SADABS. Bruker AXS Inc., Madison, Wisconsin, USA.

Bruker (2005). SAINT-Plus and SMART. Bruker AXS Inc., Madison, Wisconsin, USA.

Eshtiagh-Hosseini, H., Hassanpoor, A., Canadillas-Delgado, L. \& Mirzaei, M. (2010). Acta Cryst. E66, o1368-o1369.

Eshtiagh-Hosseini, H., Mahjoobizadeh, M. \& Mirzaei, M. (2010). Acta Cryst. E66, o2210.

Farrugia, L. J. (1997). J. Appl. Cryst. 30, 565.

Farrugia, L. J. (1999). J. Appl. Cryst. 32, 837-838.

Gellert, R. W. \& Hsu, I.-N. (1988). Acta Cryst. C44, 311-313.

Hemamalini, M. \& Fun, H.-K. (2010). Acta Cryst. E66, o1418-o1419.

Moghimi, A., Aghabozorg, H., Sheshmani, S., Kickelbick, G. \& Soleimannejad, J. (2005). Anal. Sci. 21, 141-142.

Muthiah, P. T., Balasubramani, K., Rychlewska, U. \& Plutecka, A. (2006). Acta Cryst. C62, o605-0607.

Sheldrick, G. M. (2008). Acta Cryst. A64, 112-122. 


\section{supporting information}

Acta Cryst. (2010). E66, o2996 [https://doi.org/10.1107/S160053681004345X]

\section{Acridinium 2-hydroxybenzoate}

\section{Hossein Eshtiagh-Hosseini, Azam Hassanpoor, Masoud Mirzaei and Ali R. Salimi}

\section{S1. Comment}

Molecular self-association involves the spontaneous association of molecules into stable aggregates, joined by ionpairing, hydrogen bonding, $\pi-\pi$ stacking and donor-acceptor intractions (Moghimi et al., 2005). Our research group recently focused on the syntheses as suitable ligands in the synthesis of metal-organic framework. For example, ion pairs have been reported between pyrazine-2,3-dicarboxylic acid with 2,4,6-triamino-1,3,5-triazin (Eshtiagh-Hosseini, Hassanpoor et al., 2010) and 4-hydroxy pyridine-2,6-dicarboxylic acid bearing 2-amino pyrimidine (Eshtiagh-Hosseini, Mahjoobizadeh et al., 2010). Salicylic acid is important in biological systems thus there have been several attempts to prepare proton-transfer compounds involving $\mathrm{H}_{2}$ sal with various organic bases such as 2-amino pyridine (Gellert \& Hsu, 1988), 2-amino-4,6-dimethyl primidine (Muthiah et al., 2006) and 2-amino-5-chloroprimidine (Hemamalini \& Fun, 2010). In this work, we reported a new proton-transfer compound obtained from salicylic acid $\left(\mathrm{H}_{2} \mathrm{sal}\right)$ as a proton donor and acridine (acr) as an acceptor in which acridinium $\mathrm{N}$ atom is protonated and carboxylic group of salicilic acid is deprotonated. The molecular structure of $\mathbf{I}$, is shown in Fig. 1. The crystal structure is stabilized by a network of intermolecular $\mathrm{N}-\mathrm{H} \cdots \mathrm{O}$ and $\mathrm{C}-\mathrm{H} \cdots \mathrm{O}$ hydrogen bonds with $\mathrm{H} \cdots \mathrm{A}$ distance ranging from 1.55 (2) to 2.49 (2) $\AA$ (Table 1). Furthermore, in the crystalline network there is an intramolecular $\mathrm{O}-\mathrm{H} \cdots \mathrm{O}$ hydrogen bond between phenolic $\mathrm{OH}$ and the carboxyl group (Fig. 2). In the crystal structure, $\mathrm{C}-\mathrm{H} \cdots \pi$ interactions (Table 1) $[\mathrm{Cg} 1$ is the centroid of $\mathrm{C} 2-\mathrm{C} 7$ benzene ring of $\mathrm{H}_{2} \mathrm{sal}$ ] may further stabilize the structure. Above-mentioned van der Waals interactions lead to the formation and then expansion of a proton-transfer ligand.

\section{S2. Experimental}

By refluxing $0.14 \mathrm{mmol}(0.025 \mathrm{~g}) \mathrm{H}_{2} \mathrm{sal}$ and $0.14 \mathrm{mmol}(0.025 \mathrm{~g})$ Acr in $15 \mathrm{ml}$ water for $3 \mathrm{~h}$ at $353 \mathrm{~K}$, an orange solution was obtained. This solution gave orange needle-like crystal of the title compound after slow evaporation of the solvent at R.T.

\section{S3. Refinement}

$\mathrm{H} 1$ and $\mathrm{H} 3-\mathrm{H} 7$ atoms were positioned from Fourier map and other $\mathrm{H}$ atoms were positioned geometrically and allowed to ride during refinement isotropically. $\mathrm{C}-\mathrm{H}$ distances are $0.93 \AA$ for $\mathrm{C}\left(s p^{2}\right)$ and and $U_{\text {iso }}=\mathrm{p} U_{\text {eq }}$ (parent atom) $[\mathrm{p}=1.2$ for $\left.\mathrm{C}\left(s p^{2}\right)\right]$. 


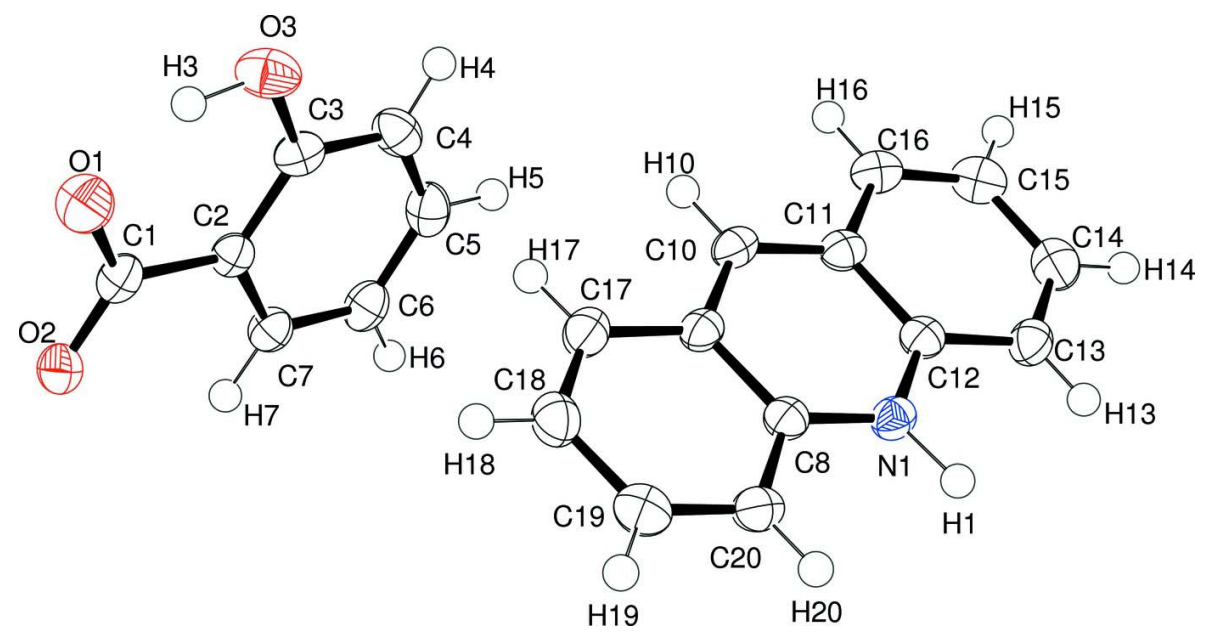

Figure 1

Schematic representation of asymmetric units of the title compound.

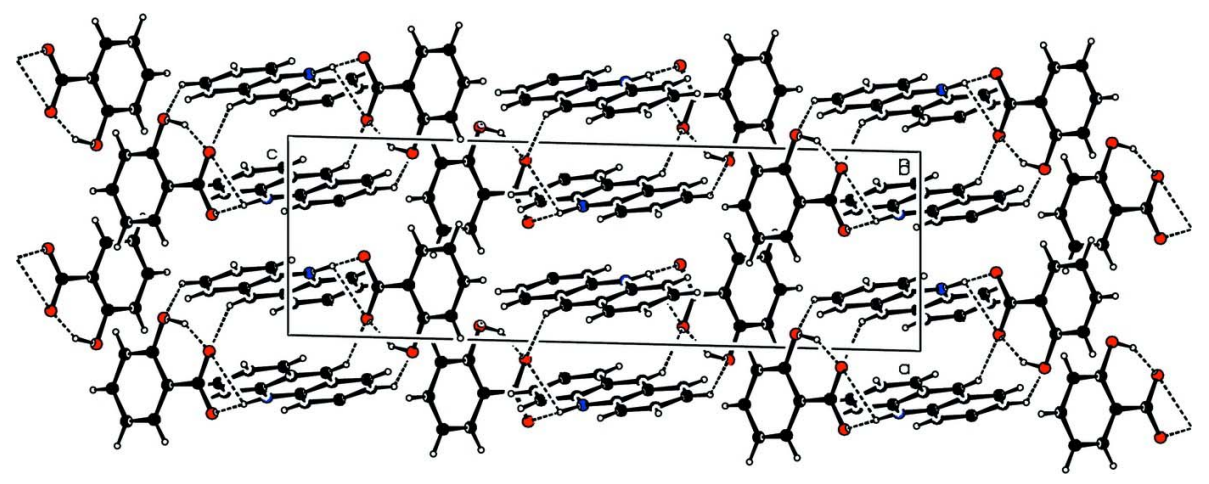

Figure 2

Molecular packing of the title compound with hydrogen bonding shown as dashed lines.

Acridinium 2-hydroxybenzoate

Crystal data

$\mathrm{C}_{13} \mathrm{H}_{10} \mathrm{~N}^{+} \cdot \mathrm{C}_{7} \mathrm{H}_{5} \mathrm{O}_{3}^{-}$

$M_{r}=317.33$

Monoclinic, $P 2{ }_{1} / c$

Hall symbol: -P2ybc

$a=7.128(3) \AA$

$b=9.472(3) \AA$

$c=22.637(9) \AA$

$\beta=91.449(10)^{\circ}$

$V=1527.9(10) \AA^{3}$

$Z=4$

\section{Data collection}

Bruker SMART APEXII CCD area-detector diffractometer

Radiation source: fine-focus sealed tube Graphite monochromator $\varphi$ and $\omega$ scans
$F(000)=664$

$D_{\mathrm{x}}=1.379 \mathrm{Mg} \mathrm{m}^{-3}$

Mo $K \alpha$ radiation, $\lambda=0.71073 \AA$

Cell parameters from 1285 reflections

$\theta=2-25^{\circ}$

$\mu=0.09 \mathrm{~mm}^{-1}$

$T=100 \mathrm{~K}$

Prism, light-orange

$0.30 \times 0.25 \times 0.10 \mathrm{~mm}$

Absorption correction: multi-scan

$$
\text { (SADABS; Bruker, 2001) }
$$

$T_{\min }=0.973, T_{\max }=0.991$

10437 measured reflections

4488 independent reflections

3161 reflections with $I>2 \sigma(I)$ 


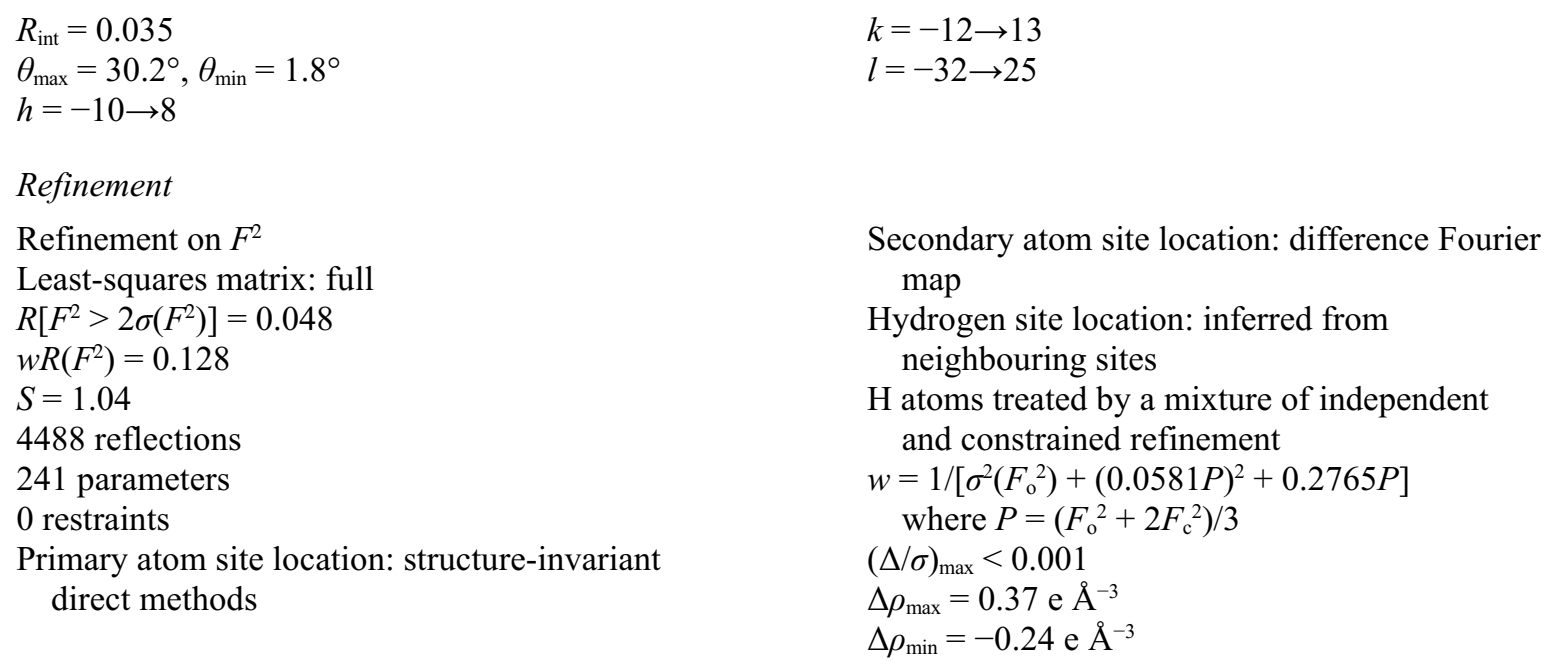

Special details

Geometry. All e.s.d.'s (except the e.s.d. in the dihedral angle between two 1.s. planes) are estimated using the full covariance matrix. The cell e.s.d.'s are taken into account individually in the estimation of e.s.d.'s in distances, angles and torsion angles; correlations between e.s.d.'s in cell parameters are only used when they are defined by crystal symmetry. An approximate (isotropic) treatment of cell e.s.d.'s is used for estimating e.s.d.'s involving l.s. planes.

Refinement. Refinement of $F^{2}$ against ALL reflections. The weighted $R$-factor $w R$ and goodness of fit $S$ are based on $F^{2}$, conventional $R$-factors $R$ are based on $F$, with $F$ set to zero for negative $F^{2}$. The threshold expression of $F^{2}>\sigma\left(F^{2}\right)$ is used only for calculating $R$-factors (gt) etc. and is not relevant to the choice of reflections for refinement. $R$-factors based on $F^{2}$ are statistically about twice as large as those based on $F$, and $R$ - factors based on ALL data will be even larger.

Fractional atomic coordinates and isotropic or equivalent isotropic displacement parameters $\left(\AA^{2}\right)$

\begin{tabular}{|c|c|c|c|c|}
\hline & $x$ & $y$ & $z$ & $U_{\text {iso }} * / U_{\text {eq }}$ \\
\hline $\mathrm{C} 1$ & $0.74651(19)$ & $0.32545(14)$ & $0.35776(6)$ & $0.0186(3)$ \\
\hline $\mathrm{C} 2$ & $0.73287(18)$ & $0.43221(14)$ & $0.30953(6)$ & $0.0163(3)$ \\
\hline $\mathrm{C} 3$ & $0.89699(18)$ & $0.48889(14)$ & $0.28532(6)$ & 0.0183 \\
\hline $\mathrm{C} 4$ & $0.8833(2)$ & $0.59342(15)$ & $0.24213(7)$ & 0.0218 \\
\hline $\mathrm{C} 5$ & $0.7091(2)$ & $0.63934(16)$ & $0.22190(7)$ & 0.0228 \\
\hline C6 & $0.5458(2)$ & $0.58278(16)$ & $0.24467(6)$ & $0.0212(3)$ \\
\hline $\mathrm{C} 7$ & $0.5587(2)$ & $0.47998(15)$ & $0.28815(6)$ & $0.0186(3)$ \\
\hline $\mathrm{C} 8$ & $0.70546(18)$ & $0.32647(15)$ & $0.01980(6)$ & 0.0169 (3) \\
\hline $\mathrm{C} 9$ & $0.76980(18)$ & $0.40801(15)$ & $0.06919(6)$ & $0.0171(3)$ \\
\hline $\mathrm{C} 10$ & $0.81472(18)$ & $0.54997(15)$ & $0.06022(6)$ & 0.0181 \\
\hline H10 & 0.8588 & 0.6041 & 0.0919 & $0.022 *$ \\
\hline C11 & $0.79458(18)$ & $0.61143(15)$ & $0.00465(6)$ & $0.0173(3)$ \\
\hline C12 & $0.72746(17)$ & $0.52574(15)$ & $-0.04321(6)$ & $0.0171(3)$ \\
\hline C13 & 0.70495 (19) & $0.58406(16)$ & $-0.10047(6)$ & $0.0203(3)$ \\
\hline H13 & 0.6604 & 0.5286 & -0.1317 & $0.024 *$ \\
\hline C14 & 0.74913 (19) & $0.72240(16)$ & $-0.10951(7)$ & 0.0236 \\
\hline H14 & 0.7341 & 0.7608 & -0.1471 & $0.028^{*}$ \\
\hline C15 & $0.8176(2)$ & $0.80885(16)$ & $-0.06248(7)$ & $0.0237(3)$ \\
\hline H15 & 0.8473 & 0.9028 & -0.0697 & $0.028 *$ \\
\hline C16 & $0.84006(19)$ & $0.75551(15)$ & $-0.00700(7)$ & 0.0207 (3) \\
\hline H16 & 0.8852 & 0.8130 & 0.0235 & $0.025 *$ \\
\hline
\end{tabular}




\begin{tabular}{lllll} 
C17 & $0.78718(19)$ & $0.34000(16)$ & $0.12514(6)$ & $0.0210(3)$ \\
H17 & 0.8286 & 0.3909 & 0.1581 & $0.025^{*}$ \\
C18 & $0.74345(19)$ & $0.20068(16)$ & $0.13068(7)$ & $0.0228(3)$ \\
H18 & 0.7544 & 0.1576 & 0.1675 & $0.027^{*}$ \\
C19 & $0.6817(2)$ & $0.12079(16)$ & $0.08115(7)$ & $0.0236(3)$ \\
H19 & 0.6535 & 0.0256 & 0.0858 & $0.028^{*}$ \\
C20 & $0.66274(19)$ & $0.18115(15)$ & $0.02653(6)$ & $0.0203(3)$ \\
H20 & 0.6224 & 0.1277 & -0.0058 & $0.024^{*}$ \\
N1 & $0.68547(15)$ & $0.38796(13)$ & $-0.03385(5)$ & $0.0176(2)$ \\
O1 & $0.90825(14)$ & $0.28524(12)$ & $0.37528(5)$ & $0.0275(3)$ \\
O2 & $0.59605(14)$ & $0.28037(11)$ & $0.37986(4)$ & $0.0220(2)$ \\
O3 & $1.06952(14)$ & $0.44374(11)$ & $0.30336(5)$ & $0.0261(3)$ \\
H1 & $0.647(3)$ & $0.324(2)$ & $-0.0700(10)$ & $0.053(6)^{*}$ \\
H3 & $1.044(3)$ & $0.376(3)$ & $0.3363(11)$ & $0.072(8)^{*}$ \\
H4 & $0.994(3)$ & $0.634(2)$ & $0.2274(8)$ & $0.034(5)^{*}$ \\
H5 & $0.699(2)$ & $0.7128(19)$ & $0.1916(8)$ & $0.029(5)^{*}$ \\
H6 & $0.421(2)$ & $0.6185(18)$ & $0.2298(8)$ & $0.028(5)^{*}$ \\
H7 & $0.447(2)$ & $0.4414(16)$ & $0.3052(7)$ & $0.018(4)^{*}$ \\
\hline
\end{tabular}

Atomic displacement parameters $\left(\AA^{2}\right)$

\begin{tabular}{lllllll}
\hline & $U^{11}$ & $U^{22}$ & $U^{33}$ & $U^{12}$ & $U^{13}$ & $U^{23}$ \\
\hline C1 & $0.0232(7)$ & $0.0150(6)$ & $0.0173(6)$ & $0.0020(5)$ & $-0.0033(5)$ & $-0.0012(5)$ \\
C2 & $0.0197(7)$ & $0.0127(6)$ & $0.0163(6)$ & $0.0005(5)$ & $-0.0011(5)$ & $-0.0011(5)$ \\
C3 & $0.0179(6)$ & $0.0156(6)$ & $0.0214(7)$ & $0.0018(5)$ & $-0.0011(5)$ & $-0.0039(5)$ \\
C4 & $0.0241(7)$ & $0.0174(7)$ & $0.0241(8)$ & $-0.0019(5)$ & $0.0046(6)$ & $-0.0002(6)$ \\
C5 & $0.0320(8)$ & $0.0176(7)$ & $0.0189(7)$ & $0.0014(6)$ & $0.0013(6)$ & $0.0006(6)$ \\
C6 & $0.0240(7)$ & $0.0203(7)$ & $0.0191(7)$ & $0.0041(6)$ & $-0.0035(5)$ & $0.0001(5)$ \\
C7 & $0.0194(7)$ & $0.0177(7)$ & $0.0185(7)$ & $0.0007(5)$ & $-0.0010(5)$ & $-0.0008(5)$ \\
C8 & $0.0128(6)$ & $0.0197(7)$ & $0.0182(7)$ & $0.0010(5)$ & $0.0010(5)$ & $-0.0011(5)$ \\
C9 & $0.0135(6)$ & $0.0209(7)$ & $0.0168(7)$ & $0.0014(5)$ & $-0.0004(5)$ & $-0.0025(5)$ \\
C10 & $0.0150(6)$ & $0.0202(7)$ & $0.0189(7)$ & $0.0011(5)$ & $-0.0016(5)$ & $-0.0043(5)$ \\
C11 & $0.0132(6)$ & $0.0185(7)$ & $0.0202(7)$ & $0.0017(5)$ & $0.0000(5)$ & $-0.0019(5)$ \\
C12 & $0.0125(6)$ & $0.0196(7)$ & $0.0193(7)$ & $0.0021(5)$ & $0.0007(5)$ & $-0.0009(5)$ \\
C13 & $0.0179(7)$ & $0.0253(7)$ & $0.0177(7)$ & $0.0016(5)$ & $-0.0006(5)$ & $-0.0011(6)$ \\
C14 & $0.0196(7)$ & $0.0282(8)$ & $0.0229(7)$ & $0.0042(6)$ & $0.0001(5)$ & $0.0055(6)$ \\
C15 & $0.0206(7)$ & $0.0188(7)$ & $0.0315(8)$ & $0.0017(5)$ & $-0.0001(6)$ & $0.0028(6)$ \\
C16 & $0.0177(7)$ & $0.0183(7)$ & $0.0262(8)$ & $0.0008(5)$ & $-0.0010(5)$ & $-0.0027(6)$ \\
C17 & $0.0191(7)$ & $0.0257(8)$ & $0.0181(7)$ & $0.0014(5)$ & $-0.0007(5)$ & $-0.0019(6)$ \\
C18 & $0.0211(7)$ & $0.0268(8)$ & $0.0206(7)$ & $0.0012(6)$ & $0.0006(5)$ & $0.0039(6)$ \\
C19 & $0.0217(7)$ & $0.0210(7)$ & $0.0282(8)$ & $-0.0005(5)$ & $0.0016(6)$ & $0.0013(6)$ \\
C20 & $0.0183(7)$ & $0.0203(7)$ & $0.0225(7)$ & $-0.0019(5)$ & $0.0003(5)$ & $-0.0033(5)$ \\
N1 & $0.0158(5)$ & $0.0195(6)$ & $0.0176(6)$ & $0.0008(4)$ & $0.0000(4)$ & $-0.0032(5)$ \\
O1 & $0.0222(5)$ & $0.0288(6)$ & $0.0311(6)$ & $0.0049(4)$ & $-0.0045(4)$ & $0.0095(5)$ \\
O2 & $0.0231(5)$ & $0.0232(5)$ & $0.0197(5)$ & $-0.0012(4)$ & $-0.0018(4)$ & $0.0050(4)$ \\
O3 & $0.0178(5)$ & $0.0223(6)$ & $0.0381(7)$ & $0.0019(4)$ & $-0.0009(4)$ & $0.0022(5)$ \\
& & & & & & \\
\hline
\end{tabular}


Geometric parameters $\left(\AA,{ }^{\circ}\right)$

\begin{tabular}{|c|c|c|c|}
\hline $\mathrm{C} 1-\mathrm{O} 1$ & $1.2681(17)$ & $\mathrm{C} 11-\mathrm{C} 12$ & $1.4268(19)$ \\
\hline $\mathrm{C} 1-\mathrm{O} 2$ & $1.2688(17)$ & $\mathrm{C} 11-\mathrm{C} 16$ & $1.429(2)$ \\
\hline $\mathrm{C} 1-\mathrm{C} 2$ & $1.4895(19)$ & $\mathrm{C} 12-\mathrm{N} 1$ & $1.3568(19)$ \\
\hline $\mathrm{C} 2-\mathrm{C} 7$ & $1.3966(19)$ & $\mathrm{C} 12-\mathrm{C} 13$ & $1.414(2)$ \\
\hline $\mathrm{C} 2-\mathrm{C} 3$ & $1.4105(19)$ & $\mathrm{C} 13-\mathrm{C} 14$ & $1.364(2)$ \\
\hline $\mathrm{C} 3-\mathrm{O} 3$ & $1.3551(17)$ & $\mathrm{C} 13-\mathrm{H} 13$ & 0.9300 \\
\hline $\mathrm{C} 3-\mathrm{C} 4$ & $1.393(2)$ & $\mathrm{C} 14-\mathrm{C} 15$ & $1.420(2)$ \\
\hline $\mathrm{C} 4-\mathrm{C} 5$ & $1.382(2)$ & $\mathrm{C} 14-\mathrm{H} 14$ & 0.9300 \\
\hline $\mathrm{C} 4-\mathrm{H} 4$ & $0.943(18)$ & $\mathrm{C} 15-\mathrm{C} 16$ & $1.359(2)$ \\
\hline $\mathrm{C} 5-\mathrm{C} 6$ & $1.392(2)$ & $\mathrm{C} 15-\mathrm{H} 15$ & 0.9300 \\
\hline $\mathrm{C} 5-\mathrm{H} 5$ & $0.979(18)$ & $\mathrm{C} 16-\mathrm{H} 16$ & 0.9300 \\
\hline $\mathrm{C} 6-\mathrm{C} 7$ & $1.386(2)$ & $\mathrm{C} 17-\mathrm{C} 18$ & $1.362(2)$ \\
\hline $\mathrm{C} 6-\mathrm{H} 6$ & $1.002(17)$ & $\mathrm{C} 17-\mathrm{H} 17$ & 0.9300 \\
\hline $\mathrm{C} 7-\mathrm{H} 7$ & $0.966(16)$ & $\mathrm{C} 18-\mathrm{C} 19$ & $1.414(2)$ \\
\hline $\mathrm{C} 8-\mathrm{N} 1$ & $1.3511(18)$ & $\mathrm{C} 18-\mathrm{H} 18$ & 0.9300 \\
\hline $\mathrm{C} 8-\mathrm{C} 20$ & $1.419(2)$ & $\mathrm{C} 19-\mathrm{C} 20$ & $1.366(2)$ \\
\hline $\mathrm{C} 8-\mathrm{C} 9$ & $1.4252(19)$ & $\mathrm{C} 19-\mathrm{H} 19$ & 0.9300 \\
\hline $\mathrm{C} 9-\mathrm{C} 10$ & $1.398(2)$ & $\mathrm{C} 20-\mathrm{H} 20$ & 0.9300 \\
\hline $\mathrm{C} 9-\mathrm{C} 17$ & $1.424(2)$ & $\mathrm{N} 1-\mathrm{H} 1$ & $1.05(2)$ \\
\hline $\mathrm{C} 10-\mathrm{C} 11$ & $1.3901(19)$ & $\mathrm{O} 3-\mathrm{H} 3$ & $1.01(3)$ \\
\hline $\mathrm{C} 10-\mathrm{H} 10$ & 0.9300 & & \\
\hline $\mathrm{O} 1-\mathrm{C} 1-\mathrm{O} 2$ & $123.12(13)$ & $\mathrm{C} 12-\mathrm{C} 11-\mathrm{C} 16$ & $118.46(13)$ \\
\hline $\mathrm{O} 1-\mathrm{C} 1-\mathrm{C} 2$ & $118.37(12)$ & $\mathrm{N} 1-\mathrm{C} 12-\mathrm{C} 13$ & $119.90(13)$ \\
\hline $\mathrm{O} 2-\mathrm{C} 1-\mathrm{C} 2$ & $118.51(12)$ & $\mathrm{N} 1-\mathrm{C} 12-\mathrm{C} 11$ & $119.98(12)$ \\
\hline $\mathrm{C} 7-\mathrm{C} 2-\mathrm{C} 3$ & $118.74(13)$ & $\mathrm{C} 13-\mathrm{C} 12-\mathrm{C} 11$ & $120.12(13)$ \\
\hline $\mathrm{C} 7-\mathrm{C} 2-\mathrm{C} 1$ & $121.00(12)$ & $\mathrm{C} 14-\mathrm{C} 13-\mathrm{C} 12$ & $119.43(13)$ \\
\hline $\mathrm{C} 3-\mathrm{C} 2-\mathrm{C} 1$ & $120.25(12)$ & $\mathrm{C} 14-\mathrm{C} 13-\mathrm{H} 13$ & 120.3 \\
\hline $\mathrm{O} 3-\mathrm{C} 3-\mathrm{C} 4$ & $118.87(13)$ & $\mathrm{C} 12-\mathrm{C} 13-\mathrm{H} 13$ & 120.3 \\
\hline $\mathrm{O} 3-\mathrm{C} 3-\mathrm{C} 2$ & $121.19(13)$ & $\mathrm{C} 13-\mathrm{C} 14-\mathrm{C} 15$ & $121.19(14)$ \\
\hline $\mathrm{C} 4-\mathrm{C} 3-\mathrm{C} 2$ & $119.94(13)$ & $\mathrm{C} 13-\mathrm{C} 14-\mathrm{H} 14$ & 119.4 \\
\hline $\mathrm{C} 5-\mathrm{C} 4-\mathrm{C} 3$ & $120.18(13)$ & $\mathrm{C} 15-\mathrm{C} 14-\mathrm{H} 14$ & 119.4 \\
\hline $\mathrm{C} 5-\mathrm{C} 4-\mathrm{H} 4$ & $120.2(11)$ & $\mathrm{C} 16-\mathrm{C} 15-\mathrm{C} 14$ & $120.57(14)$ \\
\hline $\mathrm{C} 3-\mathrm{C} 4-\mathrm{H} 4$ & $119.6(11)$ & $\mathrm{C} 16-\mathrm{C} 15-\mathrm{H} 15$ & 119.7 \\
\hline $\mathrm{C} 4-\mathrm{C} 5-\mathrm{C} 6$ & $120.56(14)$ & $\mathrm{C} 14-\mathrm{C} 15-\mathrm{H} 15$ & 119.7 \\
\hline $\mathrm{C} 4-\mathrm{C} 5-\mathrm{H} 5$ & $120.5(10)$ & $\mathrm{C} 15-\mathrm{C} 16-\mathrm{C} 11$ & $120.24(14)$ \\
\hline $\mathrm{C} 6-\mathrm{C} 5-\mathrm{H} 5$ & $119.0(10)$ & $\mathrm{C} 15-\mathrm{C} 16-\mathrm{H} 16$ & 119.9 \\
\hline $\mathrm{C} 7-\mathrm{C} 6-\mathrm{C} 5$ & $119.50(14)$ & $\mathrm{C} 11-\mathrm{C} 16-\mathrm{H} 16$ & 119.9 \\
\hline $\mathrm{C} 7-\mathrm{C} 6-\mathrm{H} 6$ & $121.3(10)$ & $\mathrm{C} 18-\mathrm{C} 17-\mathrm{C} 9$ & $120.34(13)$ \\
\hline $\mathrm{C} 5-\mathrm{C} 6-\mathrm{H} 6$ & $119.2(10)$ & $\mathrm{C} 18-\mathrm{C} 17-\mathrm{H} 17$ & 119.8 \\
\hline $\mathrm{C} 6-\mathrm{C} 7-\mathrm{C} 2$ & $121.07(13)$ & $\mathrm{C} 9-\mathrm{C} 17-\mathrm{H} 17$ & 119.8 \\
\hline $\mathrm{C} 6-\mathrm{C} 7-\mathrm{H} 7$ & $120.7(9)$ & $\mathrm{C} 17-\mathrm{C} 18-\mathrm{C} 19$ & $120.86(14)$ \\
\hline $\mathrm{C} 2-\mathrm{C} 7-\mathrm{H} 7$ & $118.2(9)$ & $\mathrm{C} 17-\mathrm{C} 18-\mathrm{H} 18$ & 119.6 \\
\hline $\mathrm{N} 1-\mathrm{C} 8-\mathrm{C} 20$ & $119.78(12)$ & $\mathrm{C} 19-\mathrm{C} 18-\mathrm{H} 18$ & 119.6 \\
\hline $\mathrm{N} 1-\mathrm{C} 8-\mathrm{C} 9$ & $119.74(13)$ & $\mathrm{C} 20-\mathrm{C} 19-\mathrm{C} 18$ & $121.03(14)$ \\
\hline $\mathrm{C} 20-\mathrm{C} 8-\mathrm{C} 9$ & $120.47(13)$ & $\mathrm{C} 20-\mathrm{C} 19-\mathrm{H} 19$ & 119.5 \\
\hline
\end{tabular}




$\begin{array}{ll}\mathrm{C} 10-\mathrm{C} 9-\mathrm{C} 17 & 123.32(13) \\ \mathrm{C} 10-\mathrm{C} 9-\mathrm{C} 8 & 118.52(13) \\ \mathrm{C} 17-\mathrm{C} 9-\mathrm{C} 8 & 118.15(13) \\ \mathrm{C} 11-\mathrm{C} 10-\mathrm{C} 9 & 121.02(13) \\ \mathrm{C} 11-\mathrm{C} 10-\mathrm{H} 10 & 119.5 \\ \mathrm{C} 9-\mathrm{C} 10-\mathrm{H} 10 & 119.5 \\ \mathrm{C} 10-\mathrm{C} 11-\mathrm{C} 12 & 118.28(13) \\ \mathrm{C} 10-\mathrm{C} 11-\mathrm{C} 16 & 123.25(13) \\ & \\ \mathrm{O} 1-\mathrm{C} 1-\mathrm{C} 2-\mathrm{C} 7 & 179.63(13) \\ \mathrm{O} 2-\mathrm{C} 1-\mathrm{C} 2-\mathrm{C} 7 & -1.2(2) \\ \mathrm{O} 1-\mathrm{C} 1-\mathrm{C} 2-\mathrm{C} 3 & -1.5(2) \\ \mathrm{O} 2-\mathrm{C} 1-\mathrm{C} 2-\mathrm{C} 3 & 177.62(12) \\ \mathrm{C} 7-\mathrm{C} 2-\mathrm{C} 3-\mathrm{O} 3 & -178.30(12) \\ \mathrm{C} 1-\mathrm{C} 2-\mathrm{C} 3-\mathrm{O} 3 & 2.8(2) \\ \mathrm{C} 7-\mathrm{C} 2-\mathrm{C} 3-\mathrm{C} 4 & 1.8(2) \\ \mathrm{C} 1-\mathrm{C} 2-\mathrm{C} 3-\mathrm{C} 4 & -177.04(13) \\ \mathrm{O} 3-\mathrm{C} 3-\mathrm{C} 4-\mathrm{C} 5 & 178.54(13) \\ \mathrm{C} 2-\mathrm{C} 3-\mathrm{C} 4-\mathrm{C} 5 & -1.6(2) \\ \mathrm{C} 3-\mathrm{C} 4-\mathrm{C} 5-\mathrm{C} 6 & 0.5(2) \\ \mathrm{C} 4-\mathrm{C} 5-\mathrm{C} 6-\mathrm{C} 7 & 0.2(2) \\ \mathrm{C} 5-\mathrm{C} 6-\mathrm{C} 7-\mathrm{C} 2 & 0.0(2) \\ \mathrm{C} 3-\mathrm{C} 2-\mathrm{C} 7-\mathrm{C} 6 & -1.1(2) \\ \mathrm{C} 1-\mathrm{C} 2-\mathrm{C} 7-\mathrm{C} 6 & 177.80(13) \\ \mathrm{N} 1-\mathrm{C} 8-\mathrm{C} 9-\mathrm{C} 10 & -1.41(18) \\ \mathrm{C} 20-\mathrm{C} 8-\mathrm{C} 9-\mathrm{C} 10 & 178.42(12) \\ \mathrm{N} 1-\mathrm{C} 8-\mathrm{C} 9-\mathrm{C} 17 & 179.33(12) \\ \mathrm{C} 20-\mathrm{C} 8-\mathrm{C} 9-\mathrm{C} 17 & -0.84(18) \\ \mathrm{C} 17-\mathrm{C} 9-\mathrm{C} 10-\mathrm{C} 11 & -179.78(12) \\ \mathrm{C} 8-\mathrm{C} 9-\mathrm{C} 10-\mathrm{C} 11 & 1.00(19) \\ \mathrm{C} 9-\mathrm{C} 10-\mathrm{C} 11-\mathrm{C} 12 & -0.26(19) \\ \mathrm{C} 9-\mathrm{C} 10-\mathrm{C} 11-\mathrm{C} 16 & -179.41(13) \\ & \\ & \\ & \end{array}$

$\begin{array}{ll}\mathrm{C} 18-\mathrm{C} 19-\mathrm{H} 19 & 119.5 \\ \mathrm{C} 19-\mathrm{C} 20-\mathrm{C} 8 & 119.13(13) \\ \mathrm{C} 19-\mathrm{C} 20-\mathrm{H} 20 & 120.4 \\ \mathrm{C} 8-\mathrm{C} 20-\mathrm{H} 20 & 120.4 \\ \mathrm{C} 8-\mathrm{N} 1-\mathrm{C} 12 & 122.44(12) \\ \mathrm{C} 8-\mathrm{N} 1-\mathrm{H} 1 & 118.3(12) \\ \mathrm{C} 12-\mathrm{N} 1-\mathrm{H} 1 & 119.1(12) \\ \mathrm{C} 3-\mathrm{O} 3-\mathrm{H} 3 & 104.2(14)\end{array}$

$\mathrm{C} 10-\mathrm{C} 11-\mathrm{C} 12-\mathrm{N} 1 \quad-0.11(18)$

$\mathrm{C} 16-\mathrm{C} 11-\mathrm{C} 12-\mathrm{N} 1 \quad 179.08(12)$

$\mathrm{C} 10-\mathrm{C} 11-\mathrm{C} 12-\mathrm{C} 13 \quad-179.99(12)$

$\mathrm{C} 16-\mathrm{C} 11-\mathrm{C} 12-\mathrm{C} 13 \quad-0.79(18)$

$\mathrm{N} 1-\mathrm{C} 12-\mathrm{C} 13-\mathrm{C} 14 \quad-179.43(12)$

$\mathrm{C} 11-\mathrm{C} 12-\mathrm{C} 13-\mathrm{C} 14 \quad 0.44$ (19)

$\mathrm{C} 12-\mathrm{C} 13-\mathrm{C} 14-\mathrm{C} 15 \quad 0.1(2)$

$\mathrm{C} 13-\mathrm{C} 14-\mathrm{C} 15-\mathrm{C} 16 \quad-0.3$ (2)

$\mathrm{C} 14-\mathrm{C} 15-\mathrm{C} 16-\mathrm{C} 11 \quad-0.1(2)$

$\mathrm{C} 10-\mathrm{C} 11-\mathrm{C} 16-\mathrm{C} 15 \quad 179.77$ (13)

$\mathrm{C} 12-\mathrm{C} 11-\mathrm{C} 16-\mathrm{C} 15 \quad 0.62(19)$

$\mathrm{C} 10-\mathrm{C} 9-\mathrm{C} 17-\mathrm{C} 18 \quad-179.08(13)$

$\mathrm{C} 8-\mathrm{C} 9-\mathrm{C} 17-\mathrm{C} 18 \quad 0.14$ (19)

$\mathrm{C} 9-\mathrm{C} 17-\mathrm{C} 18-\mathrm{C} 19 \quad 0.5$ (2)

$\mathrm{C} 17-\mathrm{C} 18-\mathrm{C} 19-\mathrm{C} 20 \quad-0.5(2)$

$\mathrm{C} 18-\mathrm{C} 19-\mathrm{C} 20-\mathrm{C} 8 \quad-0.2(2)$

$\mathrm{N} 1-\mathrm{C} 8-\mathrm{C} 20-\mathrm{C} 19 \quad-179.31(12)$

$\mathrm{C} 9-\mathrm{C} 8-\mathrm{C} 20-\mathrm{C} 19 \quad 0.86(19)$

$\mathrm{C} 20-\mathrm{C} 8-\mathrm{N} 1-\mathrm{C} 12 \quad-178.74(12)$

$\mathrm{C} 9-\mathrm{C} 8-\mathrm{N} 1-\mathrm{C} 12 \quad 1.09$ (19)

$\mathrm{C} 13-\mathrm{C} 12-\mathrm{N} 1-\mathrm{C} 8 \quad 179.55$ (12)

$\mathrm{C} 11-\mathrm{C} 12-\mathrm{N} 1-\mathrm{C} 8 \quad-0.32(19)$

Hydrogen-bond geometry $\left(\AA,{ }^{\circ}\right)$

\begin{tabular}{lllll}
\hline$D-\mathrm{H} \cdots A$ & $D-\mathrm{H}$ & $\mathrm{H} \cdots A$ & $D \cdots A$ & $D-\mathrm{H} \cdots A$ \\
\hline $\mathrm{N} 1-\mathrm{H} 1 \cdots \mathrm{O} 1^{\mathrm{i}}$ & $1.05(2)$ & $2.49(2)$ & $3.100(2)$ & $116.4(15)$ \\
$\mathrm{N} 1-\mathrm{H} 1 \cdots \mathrm{O} 2^{\mathrm{i}}$ & $1.05(2)$ & $1.55(2)$ & $2.5887(19)$ & $174.8(18)$ \\
$\mathrm{O} 3-\mathrm{H} 3 \cdots \mathrm{O} 1$ & $1.00(3)$ & $1.58(2)$ & $2.5141(19)$ & $153(2)$ \\
$\mathrm{C} 10-\mathrm{H} 10 \cdots \mathrm{O} 1^{\mathrm{ii}}$ & 0.93 & 2.49 & $3.294(2)$ & 145 \\
$\mathrm{C} 18-\mathrm{H} 18 \cdots \mathrm{O} 3^{i i i}$ & 0.93 & 2.46 & $3.135(2)$ & 129 \\
$\mathrm{C} 14-\mathrm{H} 14 \cdots C g 1^{\mathrm{iv}}$ & 0.93 & 2.76 & $3.644(2)$ & 159 \\
$\mathrm{C} 17-\mathrm{H} 17 \cdots C g 1$ & 0.93 & 2.91 & $3.716(2)$ & 146 \\
\hline
\end{tabular}

Symmetry codes: (i) $x,-y+1 / 2, z-1 / 2$; (ii) $-x+2, y+1 / 2,-z+1 / 2$; (iii) $-x+2, y-1 / 2,-z+1 / 2$; (iv) $x,-y+3 / 2, z-1 / 2$. 